\title{
Clinical outcomes after transcatheter aortic valve replacement in cancer survivors treated with ionizing radiation
}

\author{
Nikhil Agrawal ${ }^{1 \dagger}$, Sharma Kattel ${ }^{1 \dagger}$, Sameer Waheed ${ }^{1}$, Ankita Kapoor ${ }^{1}$, Vasvi Singh ${ }^{1,2}$, Ashutosh Sharma', \\ Brian J. Page ${ }^{1}$, Kristopher M. Attwood ${ }^{3}$, Vijay lyer ${ }^{1}$, Saraswati Pokharel ${ }^{4}$ and Umesh C. Sharma ${ }^{1,5^{*}}$
}

\begin{abstract}
Background: Improved cancer survival in patients treated with thoracic ionizing radiation (XRT) has resulted in unanticipated surge of aortic stenosis. Transcatheter aortic valve replacement (TAVR) has revolutionized the management of severe aortic stenosis. However, long-term clinical outcomes in radiation-exposed cohorts undergoing TAVR are unknown. We compared the all-cause mortality and major adverse cardiac events (MACE) in patients with prior chest XRT (C-XRT) undergoing TAVR.

Methods: This is an observational cohort study in subjects who underwent TAVR for symptomatic severe aortic stenosis from 2012 to 2017 in a tertiary care referral center. We examined the all-cause mortality and MACE using cox proportional hazard analysis to identify the clinical predictors of survival in the cohort of patients who had a history of prior C-XRT for malignancy.

Results: Of the 610 patients who underwent TAVR for symptomatic severe aortic stenosis, 75 had prior C-XRT. The majority of C-XRT patients had prior breast cancer (44\%) followed by Hodgkin's lymphoma (31\%), with the median time from XRT to TAVR of 19.0 years. During a mean follow up of 17.1 months after TAVR, all-cause mortality was $17 \%$. Those with prior C-XRT had higher all-cause mortality (XRT: 29\%; non-XRT:15\%, $p<0.01$ ) and MACE (XRT: 57\%; non-XRT: 27\%, $p<0.001$ ) after TAVR. Patients with prior XRT had a higher incidence of atrial fibrillation (XRT: 48\%; non-XRT: $2.4 \%, p<0.01$ ) and high-grade heart block (XRT: 20\%; non-XRT: 9.1\%, $p=0.007$ ) requiring pacemaker implant after TAVR. On multivariate cox proportional hazard analysis, prior XRT (HR: 2.07, $p=0.003$ ), poor renal function (HR: 1.29, $p<0.001$ ) and post-operative anemia requiring transfusion (HR: 1.16, p:0.001) were the strongest predictors of reduced survival.
\end{abstract}

Conclusions: Cancer survivors with prior C-XRT have higher incidence of all-cause mortality and MACE after TAVR. Careful patient selection and follow-up strategies are needed to improve outcomes.

Keywords: TAVR, Cancer survival, Radiation, And aortic stenosis

\section{Introduction}

Radiation therapy is an important therapeutic modality in multiple thoracic and non-thoracic cancers $[1,2]$. Chest radiation has been part of standard treatment protocol in various malignancies including lymphoma, breast, lung,

\footnotetext{
* Correspondence: sharmau@buffalo.edu

${ }^{\dagger}$ Nikhil Agrawal and Sharma Kattel contributed equally to this work. 'Department of Medicine, Division of Cardiology, Clinical \& Translational Research Center (Suite 7030), Jacob's School of Medicine and Biomedical Sciences, Buffalo, NY, USA

${ }^{5}$ Clinical \& Translational Research Center (Suite 7030), 875 Ellicott Street, Buffalo, NY 14203, USA

Full list of author information is available at the end of the article
}

and esophagus with improvement in cancer survival [3-5]. However, with increased longevity, cancer survivors face a higher rate of cardiovascular disease as a consequence of chest radiotherapy (C-XRT) [4, 6-8]. According to American Society of Clinical Oncology, cardiovascular complications tend to develop in $10-30 \%$ of patients receiving radiation therapy usually after a mean follow up of 5 to 10 years [9].

Radiation-Induced Valvular Heart Disease (RIVHD) is one of the most common late cardiac complications of CXRT that develops in about $10 \%$ of patients undergoing $\mathrm{C}$-XRT. The incidence of RIVHD increases in the second decade after radiation exposure $[2,10]$. Valvular lesions

(C) The Author(s). 2019 Open Access This article is distributed under the terms of the Creative Commons Attribution 4.0 International License (http://creativecommons.org/licenses/by/4.0/), which permits unrestricted use, distribution, and 
are more common on the left side of the heart than the right, most commonly involving the mitral and aortic valves [11]. C-XRT leads to late scarring, fibrotic thickening, retraction and calcification of the basal and medial portions of the leaflets with sparing of the leaflet tips and commissures allowing distinction from rheumatic valve disease $[2,12,13]$. Fossa and colleagues previously reported that 39\% of Hodgkin's lymphoma survivors who underwent C-XRT developed at least moderate to severe aortic stenosis in 12 years of follow up [12]. Another retrospective analysis of C-XRT patients' with Hodgkin's lymphoma showed valvular disease in $6.2 \%$ of patients after an average 22 years of observation, with aortic stenosis (AS) manifesting in more than a half of these patients [14].

In patients with radiation-associated AS (RA-AS), Transcatheter Aortic Valve Replacement (TAVR) has been suggested as a safer modality compared to surgical aortic valve replacement (SAVR) as mediastinal fibrosis and aortic calcifications that develop after radiotherapy makes a surgical approach more challenging [2]. Desai and colleagues recently reported poor clinical outcomes in patients with prior C-XRT who underwent SAVR [8]. Data regarding outcomes after TAVR in patients with CXRT are lacking, despite increasing number of patients currently being treated with transcatheter approach [15]. Therefore, we examined the long-term survival and major adverse cardiovascular events (MACE) in patients with prior C-XRT undergoing TAVR utilizing the American College of Cardiology National Cardiovascular Data Registry (STS/ACC TVT Registry).

\section{Methods}

This is an observational cohort study in patients who underwent TAVR for symptomatic severe AS at our tertiary care referral center. All clinical procedures and protocols conformed to institutional guidelines and were approved by the Institutional review board (IRB).

\section{Study population and design}

We studied 610 consecutive patients who underwent TAVR for symptomatic severe AS at our institute from January 2012 to September 2017. The study subjects were divided into 2 groups. The first group (XRT; $N=75$ ) had prior history of C-XRT for thoracic malignancy. The second group (non-XRT; $N=535$ ) had no history of C-XRT. The determination of prior C-XRT in cancer survivors was made based on chart review, or through a personal interview during their pre-TAVR evaluation visit.

\section{Clinical characteristics and quality of life}

Baseline patient characteristics including demographics, clinical symptomatology, surgical history, radiation history, laboratory, medications use, echocardiographic and pulmonary function test were obtained at the pre-TAVR evaluation visit. Procedural and peri-procedural complications and outcomes were obtained from the procedure notes and inpatient chart review. Baseline functional status was assessed using Kansas City Quality of Life Questionnaire (KCCQ-12) at their pre-procedure clinic visit. Surgical risk was assessed using Society of Thoracic Surgery (STS) risk score.

\section{Pre-TAVR echocardiography}

All patients underwent a comprehensive echocardiogram as part of the standard clinical diagnostic evaluation during their pre-procedure assessment for TAVR. Cardiac chamber measurements, left ventricular ejection fraction (LVEF), aortic valve area and LV stroke volume index (LVSVI) were obtained according to current American Society of Echocardiography recommended methods [16, 17].

\section{Follow-up of clinical outcomes}

The date of TAVR was considered as the beginning of the follow-up. Procedural and immediate postoperative data, length of intensive care unit or hospital stay and postoperative complications were retrieved from electronic medical records. All patients were routinely followed up after TAVR at 30 days and up to 1 year at our structural heart clinic. Beyond 1 year, data on all-cause mortality and MACE outcomes were obtained by reviewing shared electronic medical records with their primary care or health systems and by telephone follow-up.

The primary event was all cause mortality. Data on survival were obtained from medical record review, US Social Security Death Index or telephone follow-up. Cardiovascular mortality was defined as any death attributed to sudden cardiac arrest, myocardial infarction, arrhythmia, heart failure, or other cardiovascular causes. Major bleeding was defined as per the definitions of the International Society on Thrombosis and Hemostasis bleeding scale $[18,19]$.

Secondary events were composite end-point of MACE, defined as cardiovascular mortality, stroke, acute myocardial infarction (AMI) or revascularization and heart failure (HF) hospitalizations until the date of last follow up. The incidence of atrial fibrillation (AF) and atrioventricular $(\mathrm{AV})$ conduction abnormalities requiring permanent pacemaker (PPM) implant were obtained through individual review of medical records, including from device clinic or through follow-up with their primary care provider. Quality of Life Questionnaire (KCCQ-12) for each patient was assessed at 30 days and at 1-year follow-up after TAVR.

\section{Statistical analysis}

Categorical and continuous variables were expressed as percentage or frequency and mean \pm standard deviation (SD) respectively where appropriate. Baseline clinical and procedural characteristics were compared between 
the groups using the student's $t$ test or the Wilcoxon rank-sum test, as appropriate, for quantitative variables; and the Pearson chi-square test for categorical variables.

All the available relevant clinical, echocardiographic, laboratory and pre/post-operative variables were used in univariate cox proportional hazard analysis to determine the association with all-cause mortality. The variables that were significant $(p<0.05)$ on univariate analysis were used to construct the multivariate cox proportional hazard model. Kaplan-Meier survival curves for all-cause mortality, composite and each component of MACE free survival were performed. A $p$-value of $<0.05$ was considered statistically significant for all statistical analyses. All statistical analyses were performed using SAS statistical software, version 9.4 (SAS Institute Inc., Cary, NC).

\section{Results}

Comparison of baseline characteristics

Clinical, echocardiographic parameters and quality of life

Comparison of the baseline clinical, echocardiographic and quality of life variables are shown in Table 1. Smoking and anemia were more common and use of ACE/ ARBs was less frequent in the XRT group. Both the groups had identical surgical risk (STS score) and functional status (KCCQ-12) scores at baseline.

\section{Natural history and presence of multivalvular lesions}

The median time from C-XRT to TAVR was 19.0 years (Mean $20.1 \pm 4.9$ yrs.). Of the 75 symptomatic severe aortic stenosis patients with prior C-XRT who underwent TAVR, breast cancer (44\%) was the commonest reason for C-XRT followed by Hodgkin's lymphoma (31\%), lung cancer (15\%), non-Hodgkin's lymphoma (7\%) and others (3\%). Among breast cancer survivors who had C-XRT, $64 \%$ had left sided malignancy.

Moderate to severe aortic, mitral and tricuspid regurgitation was seen in 16.2, 20.8 and $18 \%$ of the total study subjects, respectively. The incidence of moderate-severe mitral stenosis was disproportionately higher in the XRT group.

\section{Age and sex disparities}

Females were disproportionally more frequent among the cancer survivors with prior C-XRT (XRT: 61\%, nonXRT: $45 \%, p=0.01$ ), which likely reflects the higher proportion of breast cancer survivors in the XRT group. The male population was slightly younger than females at the time of TAVR (mean age, male/female: $81.3 \pm 8.2$ vs $82.8 \pm 7.6, p=0.02)$. Mean STS score was higher in females (male/female: $8.2 \pm 4.6$ vs $9.9 \pm 5.9, p<0.0001$ ).

The incidence of AF and AV block requiring PPM were more common in females but there were no differences in all-cause mortality, MACE, major bleeding, or length of hospital stay among male or female patients.
Females subjects had lower KCCQ-12 scores at 30 days and 1-year follow-up compared to male counterparts (male/ female, mean score at 30 days: $80.88 \pm 15.88$ vs. $77.46 \pm$ $18.20, p=0.01$; at 1 year: $84.51 \pm 12.14$ vs. $81.44 \pm 14.61, p=$ 0.008 ) though there was no difference in their baseline score.

In a subgroup analysis of all females who underwent TAVR $(N=290, \mathrm{XRT}=46$; non-XRT $=244)$, females who had C-XRT were slightly younger (mean age, XRT vs. without XRT: $80.8 \pm 8.4$ vs. $83.2 \pm 7.5, p=0.04$ ).

\section{Comparison of Peri-procedural events}

Perioperative and post-operative characteristics are shown in Table 2. There were no differences in intravascular access, procedure duration or type of valves used among the groups.

The overall incidence of moderate to severe paravalvular leak post-TAVR was less than $1.5 \%$ in the entire study subjects and there were no significant differences among the groups.

\section{Post-TAVR outcomes}

The short and long-term events and quality of life measures after TAVR are shown in Table 3.

\section{Short-term outcomes}

The overall in-hospital incidences for AMI, AF, stroke and all-cause mortality were $0.5,8,1.5$, and $2.8 \%$, respectively. XRT group had higher incidence of in-hospital AF, stroke and all-cause mortality but no difference in 30 days mortality or major bleeding among the groups.

\section{Long-term outcomes}

During a mean follow up of 17.1 months (median: 13 months) post-TAVR, the all-cause mortality was $17 \%$ in the study population. Similarly, the incidence of MACE was $30 \%$ in the entire study population. There were significantly higher rates of mortality and MACE in the XRT group (XRT/non-XRT groups: Death, $29 \%$ vs $15 \%$, $p=0.004$; MACE, $54 \%$ vs $27 \%, p<0.0001$, respectively). The overall incidence of high-grade AV block requiring PPM implantation was $10.5 \%$ in all the study subjects and was disproportionally higher in XRT group (XRT/ non-XRT group: $19 \%$ vs $9 \%, p=0.001$ ).

Time to event survival analysis by Kaplan-Meier analysis showed reduced survival and increased incidence of MACE in the XRT group as shown in Fig. 1a-b. Similarly, Kaplan-Meier analysis showed increased incidence of HF and stroke but not for $\mathrm{CV}$ mortality and AMI or urgent revascularization in the XRT group as shown in Fig. 2a-d.

The results of the univariate and multivariate cox proportional hazard analysis for all-cause mortality are shown in Table 4. Prior C-XRT exposure (HR: 2.07; 95\% CI:1.24-3.31, $p=0.005$ ), poor renal function post TAVR (HR: 1.43, 95\% CI:1.11-1.85, $p=0.004$ ) and post-operative anemia requiring transfusion (HR: 1.17; 95\% CI: 1.05-1.30, $p=0.003$ ) were the strongest predictors of reduced survival. 
Table 1 Baseline characteristics of the study population

\begin{tabular}{|c|c|c|c|c|}
\hline Variables $^{\mathrm{a}}$ & All subjects $(n=610)$ & Non- XRT Group $(n=535)$ & C-XRT Group $(n=75)$ & $P$ value \\
\hline \multicolumn{5}{|l|}{ Clinical, demographic, and symptom variables } \\
\hline Age (years) & $82.00 \pm 7.99$ & $82.67 \pm 7.98$ & $81.64 \pm 7.81$ & 0.21 \\
\hline Gender (M/F) & $320 / 290$ & $291 / 244$ & $29 / 46$ & 0.013 \\
\hline Body Mass Index, $\mathrm{kg} / \mathrm{m}^{2}$ & $27.99 \pm 6.02$ & $28.11 \pm 5.98$ & $27.14 \pm 6.32$ & 0.10 \\
\hline Race & & & & 0.38 \\
\hline White & $587(96.4)$ & $516(96.6)$ & $71(94.7)$ & \\
\hline Blacks & $16(2.6)$ & $12(2.2)$ & $4(5.3)$ & \\
\hline Hispanics & $3(0.5)$ & $3(0.6)$ & & \\
\hline Others & $3(0.5)$ & $3(0.6)$ & & \\
\hline Hypertension & $542(88.8)$ & $476(88.9)$ & $66(88)$ & 0.84 \\
\hline Diabetes mellitus & $205(33.60)$ & $176(32.5)$ & $31(41.3)$ & 0.15 \\
\hline Hyperlipidemia & $355(58.19)$ & $307(57.3)$ & $48(64)$ & 0.31 \\
\hline Smoking history & $304(49.83)$ & $251(46.9)$ & $53(70.6)$ & $<0.0001$ \\
\hline Prior stroke & $63(10.33)$ & $53(9.9)$ & $10(13.33)$ & 0.41 \\
\hline COPD & $263(43.11)$ & $229(42.8)$ & $34(45.33)$ & 0.7 \\
\hline$C A D$ & $357(58.52)$ & $307(57.3)$ & $50(66.67)$ & 0.13 \\
\hline 3-Vessel CAD & $170(27.9)$ & $147(27.5)$ & $23(30.7)$ & 0.58 \\
\hline End-stage renal disease & $31(5.08)$ & $26(4.8)$ & $5(6.67)$ & 0.57 \\
\hline Atrial fibrillation & $249(40.81)$ & $223(41.6)$ & $26(34.6)$ & 0.26 \\
\hline PAD & $224(36.72)$ & $201(37.57)$ & $23(30.6)$ & 0.3 \\
\hline Prior $\mathrm{CABG}$ & $192(31.47)$ & $166(31.0)$ & $26(34.67)$ & 0.51 \\
\hline Pacemaker Implant History & 100 (16.39) & $82(15.33)$ & $18(24.00)$ & 0.06 \\
\hline ICM (EF < 50\% with CAD) & $111(18.19)$ & $97(18.1)$ & $14(18.67)$ & 0.87 \\
\hline NYHA Class & & & & 0.36 \\
\hline I & $4(0.7)$ & $4(0.7)$ & & \\
\hline$\|$ & $76(12.5)$ & $65(12.1)$ & $11(14.7)$ & \\
\hline III & $462(75.7)$ & $410(76.6)$ & $52(69.3)$ & \\
\hline IV & $68(11.1)$ & $56(10.5)$ & $12(16.0)$ & \\
\hline Syncope & $38(6.2)$ & $33(6.17)$ & $5(6.67)$ & 0.8 \\
\hline Angina & $194(31.8)$ & $172(32.15)$ & $22(29.33)$ & 0.69 \\
\hline Dyspnea on exertion & $555(91)$ & $487(91.03)$ & $68(90.67)$ & 0.83 \\
\hline FEV1 (\% of predicted) & $75.14 \pm 24.6$ & $75.16 \pm 24.81$ & $75.02 \pm 23.73$ & 0.96 \\
\hline STS score, Mean & $9.03 \pm 5.4$ & $9.02 \pm 5.41$ & $9.11 \pm 5.06$ & 0.7 \\
\hline STS Score, Median (Interquartile) & $8.1(5.3-11)$ & $8.1(5.3-11)$ & $8.1(5.4-11)$ & \\
\hline KCCQ12 Index & $36.4 \pm 19.6$ & $36.80 \pm 19.78$ & $33.72 \pm 18.2$ & 0.2 \\
\hline \multicolumn{5}{|l|}{ Laboratory data } \\
\hline Creatinine, mg/dl & $1.4 \pm 1.1$ & $1.38 \pm 1.12$ & $1.39 \pm 1.10$ & 0.92 \\
\hline Glomerular filtration rate, $\mathrm{mL} / \mathrm{min}$ per $1.73 \mathrm{~m}^{2}$ & $53.6 \pm 22.3$ & $53.68 \pm 22.45$ & $52.79 \pm 21.4$ & 0.74 \\
\hline Hemoglobin, mg/dl & $12 \pm 1.8$ & $12.07 \pm 1.80$ & $11.60 \pm 1.49$ & 0.03 \\
\hline \multicolumn{5}{|l|}{ Medications } \\
\hline Aspirin & $402(66)$ & $354(66.1)$ & $48(73.8)$ & 0.26 \\
\hline Beta-blockers & $491(80.5)$ & $433(80.9)$ & $58(81.6)$ & 1 \\
\hline ACE-I/ARBS & $215(35.2)$ & $200(37.3)$ & $15(23.0)$ & 0.02 \\
\hline Statins & $356(58.4)$ & $307(75.3)$ & 49 (65.3) & 0.21 \\
\hline
\end{tabular}


Table 1 Baseline characteristics of the study population (Continued)

\begin{tabular}{|c|c|c|c|c|}
\hline Variables $^{a}$ & All subjects $(n=610)$ & Non- XRT Group $(n=535)$ & C-XRT Group $(n=75)$ & $P$ value \\
\hline \multicolumn{5}{|l|}{ Echo Parameters } \\
\hline LVEF & $54.6 \pm 13$ & $54.46 \pm 13.1$ & $55.65 \pm 12.40$ & 0.45 \\
\hline AVA (cm2) & $0.65 \pm 0.2$ & $0.65 \pm 0.2$ & $0.63 \pm 0.2$ & 0.36 \\
\hline Mean AV gradient $(\mathrm{mm} \mathrm{Hg})$ & $41.14 \pm 15.28$ & $40.87 \pm 15.48$ & $43.06 \pm 13.67$ & 0.24 \\
\hline Peak AV gradient (mm Hg) & $67.04 \pm 24.72$ & $66.90 \pm 25.02$ & $68.05 \pm 22.61$ & 0.7 \\
\hline LV-SVI (ml/m2) & $37.14 \pm 13.50$ & $36.72 \pm 13.49$ & $40.11 \pm 13.30$ & 0.04 \\
\hline Abnormal LV-SVI $(<35 \mathrm{~mL} / \mathrm{m} 2)$ & $294(48.2)$ & $271(50.65)$ & $23(30.67)$ & 0.001 \\
\hline RVSP $(m m ~ H g)$ & $47.24 \pm 14$ & $47.11 \pm 14.13$ & $48.18 \pm 13.07$ & 0.53 \\
\hline Moderate-Severe AR & $99(16.22)$ & $85(15.89)$ & $14(18.66)$ & 0.52 \\
\hline Moderate-Severe MR & $127(20.81)$ & $106(19.81)$ & $21(28.00)$ & 0.21 \\
\hline Moderate-Severe TR & $110(18.03)$ & $95(17.76)$ & $15(20.00)$ & 0.48 \\
\hline Moderate-Severe MS & $45(7.37)$ & $34(6.36)$ & $11(14.67)$ & 0.01 \\
\hline
\end{tabular}

C-XRT indicates mediastinal radiation therapy

$C A D$ coronary artery disease, $C O P D$ chronic obstructive pulmonary disease, $P A D$ periphery artery disease, $C A B G$ coronary artery bypass grafting, ICM ischemic cardiomyopathy, FEV1 forced expiratory volume at $1 \mathrm{~s}$, STS Society of Thoracic Surgeons, KCCQ12 Kansas City Cardiomyopathy Questionnaire, ACE-I/ARBs Angiotensin-converting enzyme inhibitors/ Angiotensin II receptor, LVEF left ventricular ejection fraction, AVA aortic valve area, AV aortic valve, LV-SVI left ventricular stroke volume index, RVSP right ventricular systolic pressure, $A R$ aortic regurgitation, MR mitral regurgitation, TR tricuspid regurgitation, MS mitral stenosis

${ }^{a}$ Values are expressed as number (percentage) or mean \pm SD

\section{Quality of life outcomes}

Nearly $84 \%$ of the subjects were able to leave the hospital on average 6 days after TAVR. There was an increased trend toward a longer in-hospital stay in XRT group, however this didn't reach statistical significance. Functional status as measured by KCCQ-12 at baseline, 30 days and 1 year after TAVR were similar among groups as shown in Additional file 1: Figure S1. When compared in all subjects, those who were surviving had higher recovery of KCCQ-12 at 30 days, however no difference at 1 year as shown in Additional file 2: Figure S2.

\section{Subgroup analysis within the XRT group Age and sex disparities in XRT group}

In subgroup analysis of the XRT group $(N=75)$, there was no difference in the mean STS score among male or female subjects. Angina was more common in female subjects (male/female: $13.7 \%$ vs. $39.13 \%, p=0.02$ ).

Table 2 Perioperative and Postoperative Characteristics of the Study Population

\begin{tabular}{|c|c|c|c|c|}
\hline Variable $^{a}$ & All subjects $(n=610)$ & Non- XRT Group $(n=535)$ & C-XRT Group $(n=75)$ & $P$ value \\
\hline \multicolumn{5}{|l|}{ TAVR Access/duration } \\
\hline Femoral Access & $535(87.7)$ & $472(88.22)$ & $63(84.0)$ & 0.34 \\
\hline Median Procedure duration (hrs:mins/ interquartile) & $2: 17(1: 40-2: 48)$ & $2: 16(1: 04-3: 28)$ & $2: 23(1: 29-3: 07)$ & 0.38 \\
\hline \multicolumn{5}{|l|}{ Valve types } \\
\hline Edwards Sapien & $500(81.9)$ & $438(81.87)$ & $62(82.67)$ & \multirow[t]{2}{*}{0.86} \\
\hline Core Valve & $110(18.1)$ & $97(18.13)$ & $13(17.33)$ & \\
\hline \multicolumn{5}{|l|}{ Post-procedure parameters } \\
\hline Mod-severe Paravalvular leak & $9(1.48)$ & $8(1.5)$ & $1(1.35)$ & 0.91 \\
\hline Post-procedure Hb & $9.66 \pm 1.75$ & $9.73 \pm 1.76$ & $9.12 \pm 1.6$ & 0.004 \\
\hline Post-procedure Creatinine & $1.52 \pm 1.34$ & $1.50 \pm 1.28$ & $1.64 \pm 1.7$ & 0.41 \\
\hline Creatinine at discharge & $1.3 \pm 1.1$ & $1.3 \pm 1.1$ & $1.28 \pm 1.1$ & 0.73 \\
\hline Post TAVR LVEF & $55.05 \pm 12.4$ & $54.72 \pm 12.74$ & $57.4 \pm 9.8$ & 0.081 \\
\hline Post TAVR AVA, $\mathrm{cm}^{2}$ & $1.90 \pm 0.49$ & $1.91 \pm 0.49$ & $1.87 \pm 0.5$ & 0.53 \\
\hline Post TAVR Peak gradient, mm Hg & $7.93 \pm 4.64$ & $7.94 \pm 4.59$ & $7.87 \pm 5$ & 0.9 \\
\hline Post TAVR mean gradient, $\mathrm{mm} \mathrm{Hg}$ & $3.62 \pm 2.28$ & $3.62 \pm 2.23$ & $3.64 \pm 2.6$ & 0.94 \\
\hline
\end{tabular}

C-XRT indicates mediastinal radiation exposure

$H b$ hemoglobin, $L V E F$ left ventricular ejection fraction, AVA aortic valve area

${ }^{a}$ Values are expressed as number (percentage) or mean $\pm S D$ 
Table 3 Short and long-term outcomes post-TAVR

\begin{tabular}{|c|c|c|c|c|}
\hline Outcomes $^{a}$ & All Subjects $(n=610)$ & Non- XRT Group $(n=535)$ & C-XRT Group $(n=75)$ & $P$ value \\
\hline \multicolumn{5}{|l|}{ Early Events } \\
\hline ICU LOS (hrs) & $41.9 \pm 43.1$ & $41.50 \pm 43.19$ & $44.67 \pm 43.00$ & 0.55 \\
\hline Hospital LOS (days) & $5.2 \pm 6.5$ & $5.32 \pm 6.84$ & $4.52 \pm 3.64$ & 0.31 \\
\hline In Hospital AMI & $1(0.16)$ & $1(0.19)$ & $0(0)$ & 1.0 \\
\hline In Hospital AF & 49 (8.03) & $13(2.43)$ & $36(48.00)$ & $<0.0001$ \\
\hline In Hospital stroke & $9(1.48)$ & $5(0.93)$ & $4(5.33)$ & 0.01 \\
\hline In Hospital cardiac arrest & $20(3.28)$ & $15(2.80)$ & $5(6.67)$ & 0.08 \\
\hline In Hospital Mortality & $17(2.79)$ & $12(2.24)$ & $5(6.67)$ & 0.04 \\
\hline 30-day Mortality & $17(2.79)$ & $12(2.24)$ & $5(6.67)$ & 0.04 \\
\hline Major bleed & $63(10.33)$ & $52(9.72)$ & $11(14.67)$ & 0.22 \\
\hline \multicolumn{5}{|l|}{ Long-term Events } \\
\hline $\mathrm{AMI}$ and/or urgent $\mathrm{PCl}$ & $35(5.74)$ & $31(5.79)$ & $4(5.33)$ & 1.00 \\
\hline Stroke/TIA & $36(5.90)$ & $27(5.05)$ & $9(12.00)$ & 0.03 \\
\hline HF admission & $113(18.52)$ & $90(16.82)$ & $23(30.67)$ & 0.006 \\
\hline PPM implants & $64(10.49)$ & $49(9.16)$ & $15(20.0)$ & 0.007 \\
\hline CV Mortality & $50(48.54)$ & $36(44.44)$ & $14(63.64)$ & 0.14 \\
\hline All-Cause Mortality & $103(16.89)$ & $81(15.14)$ & $22(29.33)$ & 0.004 \\
\hline MACE & $185(30.33)$ & $144(26.92)$ & $41(54.67)$ & $<0.0001$ \\
\hline \multicolumn{5}{|l|}{ Quality of Life measures } \\
\hline Home Disposition post TAVR & $513(84.10)$ & $451(84.30)$ & $62(82.67)$ & 0.73 \\
\hline $\mathrm{KCCQ} 12$ at 30 days & $79.23 \pm 17.11$ & $79.27 \pm 16.98$ & $78.96 \pm 18.20$ & 0.88 \\
\hline KCCQ12 at 1 year & $83.02 \pm 13.47$ & $82.86 \pm 13.55$ & $84.29 \pm 12.81$ & 0.44 \\
\hline
\end{tabular}

$\mathrm{C}$-XRT indicates mediastinal radiation therapy

ICU intensive care unit, LOS length of stay, $A F$ atrial Fibrillation, $A M I$ acute myocardial infarction, $P C l$ percutaneous coronary intervention, $T I A$ transient ischemic attack, HF heart failure, PPM permanent pacemaker, CV cardiovascular, MACE major cardiovascular adverse event (CV death/MI/Stroke/HF), KCCQ12 Kansas City Cardiomyopathy Questionnaire

${ }^{\mathrm{a}}$ Values are expressed as number (percentage) or mean \pm SD

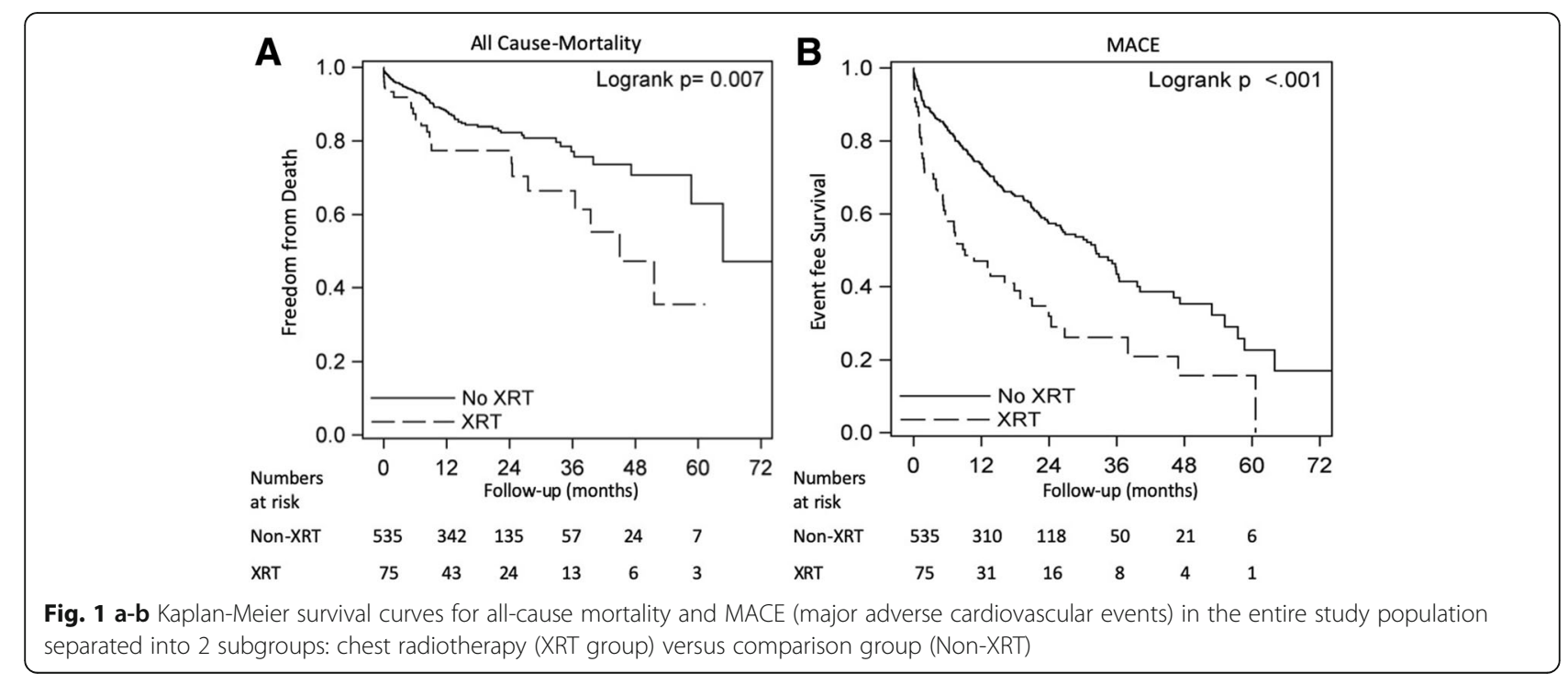



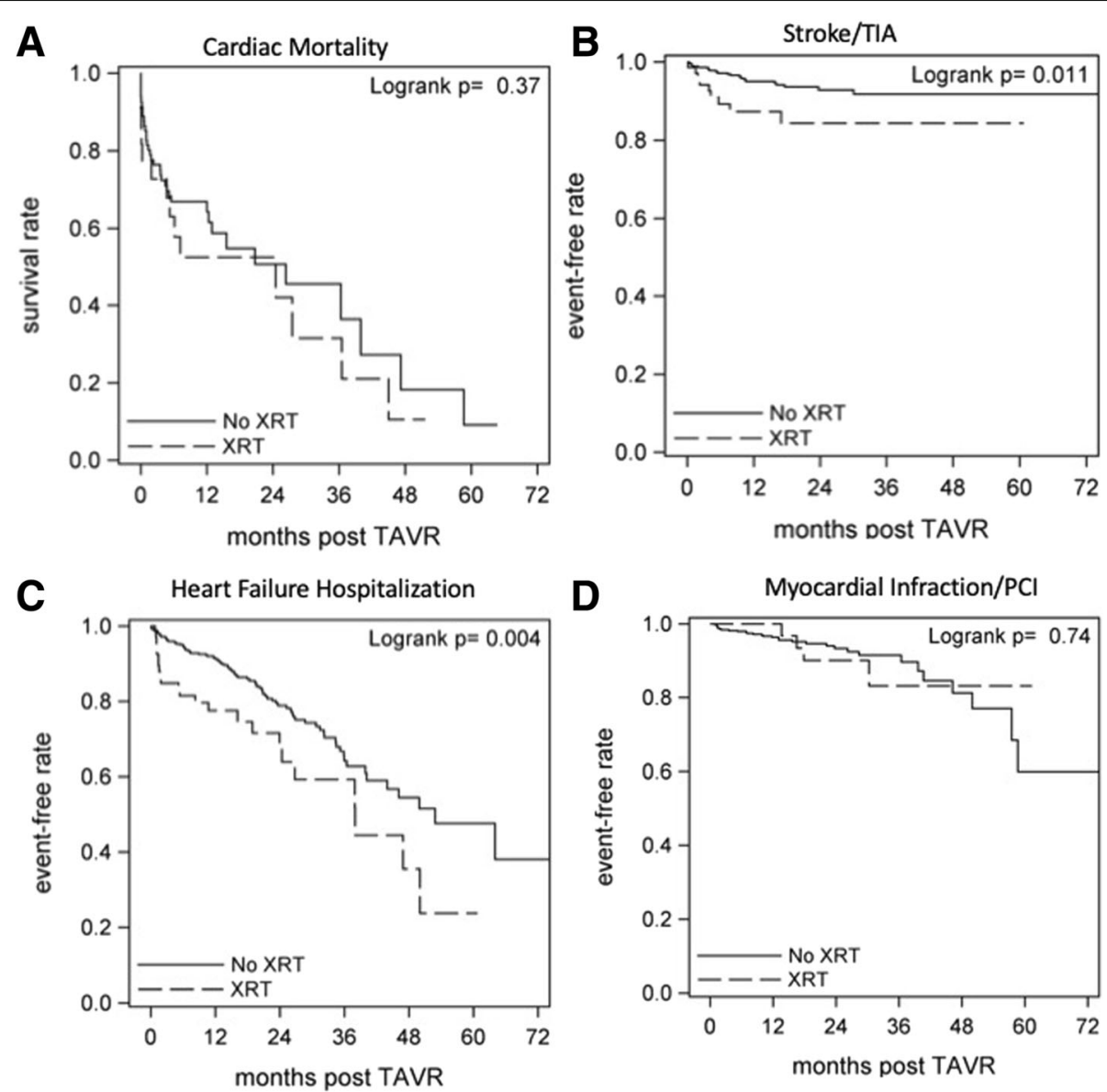

Fig. 2 a-d Kaplan-Meier curves for a cardiac mortality, $\mathbf{b}$ stroke/TIA, c heart failure hospitalization and $\mathbf{d m y o c a r d i a l}$ infraction/PCI in the entire study population separated into 2 subgroups: chest radiotherapy (XRT group) versus comparison (Non-XRT group)

Overall, $50 \%$ of the patients had prior CAD with a higher incidence in male patients (male vs. female: $82.7 \%$ vs. $56.5 \%, p=0.02$ ). All-cause mortality was higher in males compared to females (male/female:51.7\% vs $15.22 \%, p<0.001$ ), however there was no differences in 30-day mortality, MACE, KCCQ-12 among male or female subjects. After TAVR, there was an average improvement of KCCQ-12 scores by $44.5 \pm 21.02$ and $48.95 \pm 19.87$ points at 30 days and 1 year, respectively, which was similar compared to all subjects in the study.

\section{Clinical outcomes in relation to cancer types}

In cancer survivors, the all-cause mortality differed according to the cancer types, which has been summarized in the Additional file 3: Table S1. Similarly, the MACE outcomes differed according to the cancer types and were highest among Hodgkin's lymphoma patients. The incidence of stroke was also highest among Hodgkin's lymphoma followed by breast cancer, and none in other cancer survivors during the follow-up period.

\section{Discussion}

Our study demonstrates that patients who underwent TAVR with prior C-XRT for thoracic cancers have poor survival compared to the those who had undergone same procedure. Furthermore, those who had prior CXRT had less than 50\% freedom from MACE during average of 17.1 months follow-up. This is the first study evaluating the long-term outcome of TAVR in thoracic cancer survivors with prior history of C-XRT.

\section{Prognostic impact of prior chest radiation on survival}

Our data demonstrated that the patients with prior CXRT had nearly 2-fold increase in mortality after the median follow-up duration of 17 months after TAVR. The mortality differences between the XRT and control groups were discernible at the early in-hospital period and became more prominent with increasing follow-up interval. Our multivariate analysis revealed prior C-XRT, post-operative anemia requiring blood transfusion and poor renal function as the significant predictors of reduced survival. Larger multicenter studies on the longterm outcomes of RIVHD are limited. Based on a recent 
Table 4 Univariate and multivariable cox proportional hazard analysis for all-cause mortality

\begin{tabular}{|c|c|c|c|c|}
\hline \multirow[t]{2}{*}{ Variables } & \multicolumn{2}{|l|}{ Univariate analysis } & \multicolumn{2}{|l|}{ Multivariate analysis } \\
\hline & Hazard Ratio (95\% Cl) & $p$ value & Hazard Ratio $(95 \% \mathrm{Cl})$ & $p$ value \\
\hline \multicolumn{5}{|l|}{ Clinical variables } \\
\hline Prior XRT & $1.94(1.18-3.06)$ & 0.009 & $2.07(1.24-3.31)$ & 0.005 \\
\hline STS score (per unit change) & $1.04(1.01-1.07)$ & 0.001 & $1.00(0.96-1.03)$ & 0.93 \\
\hline Age (per unit change) & $0.99(0.97-1.02)$ & 0.7 & & \\
\hline Gender (Male versus Female) & $1.2(0.81-1.78)$ & 0.35 & & \\
\hline $\mathrm{BMI}\left(\mathrm{kg} / \mathrm{m}^{2}\right.$, per unit change) & $0.99(0.96-1.03)$ & 0.9 & & \\
\hline Hypertension & $1.21(0.62-2.72)$ & 0.58 & & \\
\hline Diabetes mellitus & $1.00(0.66-1.50)$ & 0.96 & & \\
\hline Hyperlipidemia & $1.54(1.02-2.37)$ & 0.03 & $1.48(0.96-2.31)$ & 0.07 \\
\hline Smoking & $1.37(0.92-2.04)$ & 0.11 & & \\
\hline Prior Stroke & $1.60(0.98-2.51)$ & 0.05 & & \\
\hline COPD & $1.49(1.01-2.20)$ & 0.04 & $1.21(0.74-2.00)$ & 0.43 \\
\hline$C A D$ & $1.29(0.86-1.97)$ & 0.21 & & \\
\hline 3 Vessel CAD & $0.85(0.53-1.32)$ & 0.49 & & \\
\hline End stage renal disease & $3.03(1.61-5.23)$ & 0.001 & $0.96(0.28-2.92)$ & 0.94 \\
\hline Peripheral artery disease & $1.24(0.82-1.84)$ & 0.29 & & \\
\hline Atrial Fibrillation & $1.30(0.87-1.92)$ & 0.18 & & \\
\hline Prior Cardiac surgery & $0.90(0.59-1.36)$ & 0.65 & & \\
\hline ICM (EF < 50\% and CAD) & $1.25(0.73-1.89)$ & 0.44 & & \\
\hline Previous $\mathrm{PCl}$ & $1.03(0.62-1.64)$ & 0.88 & & \\
\hline Prior Pacemaker & $1.24(0.74-1.99)$ & 0.38 & & \\
\hline Angina & $0.97(0.90-1.46)$ & 0.9 & & \\
\hline Syncope & $1.21(0.53-2.36)$ & 0.61 & & \\
\hline Dyspnea on exertion & $1.37(0.70-3.10)$ & 0.36 & & \\
\hline Pre-TAVR KCCQ12 score (per unit change) & $0.37(0.12-1.06)$ & 0.06 & & \\
\hline \multicolumn{5}{|l|}{ Lab /Echo Variable } \\
\hline Pre-TAVR Hb (gm, per unit change) & $0.79(0.70-0.89)$ & 0.0002 & $090(0.78-1.04)$ & 0.17 \\
\hline Creatinine (per unit change) & $1.22(1.09-1.33)$ & 0.0006 & $0.87(0.66-1.13)$ & 0.32 \\
\hline eGFR (per unit change) & $0.99(0.98-1.00)$ & 0.13 & & \\
\hline FEV1 (per unit change) & $0.98(0.98-0.99)$ & 0.01 & $0.99(0.98-1.00)$ & 0.43 \\
\hline LV EF (\%, per unit change) & $0.99(0.98-1.01)$ & 0.59 & & \\
\hline AR (per level increase in severity) & $1.26(0.71-2.24)$ & 0.06 & & \\
\hline Presence of MS & $1.80(0.96-3.12)$ & 0.06 & & \\
\hline MR (per level increase in severity) & $1.32(0.69-2.51)$ & 0.33 & & \\
\hline TR (per level increase in severity) & $0.81(0.41-1.58)$ & 0.36 & & \\
\hline AVA (cm2, per unit change) & $1.38(0.47-3.87)$ & 0.54 & & \\
\hline Mean AV gradient (mmHg, per unit change) & $0.99(0.98-1.00)$ & 0.43 & & \\
\hline Peak AV gradient (mmHg, per unit change) & $0.99(0.99-1.00)$ & 0.88 & & \\
\hline Reduced LV-SVI & $1.13(0.76-1.67)$ & 0.51 & & \\
\hline RVSP (mmHg, per unit change) & $1.01(1.00-1.02)$ & 0.01 & $1.01(0.99-1.02)$ & 0.05 \\
\hline \multicolumn{5}{|l|}{ Medications } \\
\hline Aspirin & $1.15(0.76-1.78)$ & 0.5 & & \\
\hline Beta-Blocker & $1.37(0.79-2.58)$ & 0.27 & & \\
\hline
\end{tabular}


Table 4 Univariate and multivariable cox proportional hazard analysis for all-cause mortality (Continued)

\begin{tabular}{|c|c|c|c|c|}
\hline \multirow[t]{2}{*}{ Variables } & \multicolumn{2}{|l|}{ Univariate analysis } & \multicolumn{2}{|l|}{ Multivariate analysis } \\
\hline & Hazard Ratio (95\% Cl) & $p$ value & Hazard Ratio (95\% Cl) & $p$ value \\
\hline Statins & $1.52(0.99-2.32)$ & 0.05 & & \\
\hline ACE/ARBs & $1.05(0.69-1.57)$ & 0.81 & & \\
\hline \multicolumn{5}{|l|}{ Operative and Post-Operative variables } \\
\hline Operative time (in hours, per unit change) & $1.00(0.99-1.00)$ & 0.09 & & \\
\hline TVT Access (Femoral vs others) & $1.07(0.63-1.94)$ & 0.8 & & \\
\hline Type of Valve (Edward Sapiens vs Core valve) & $0.77(0.48-1.29)$ & 0.31 & & \\
\hline Para-valvular leak (mod-severe) & $0.58(0.09-2.01)$ & 0.18 & & \\
\hline Post Op Atrial fibrillation & $1.55(0.82-2.68)$ & 0.16 & & \\
\hline Creatinine at discharge (mg, per unit change) & $1.33(1.19-1.47)$ & $<0.001$ & $1.43(1.11-1.85)$ & 0.004 \\
\hline Post procedure $\mathrm{Hb}$ (gm, per unit change) & $0.82(0.72-0.92)$ & 0.001 & $1.03(0.87-1.23)$ & 0.66 \\
\hline Per units' blood transfused & $1.22(1.12-1.32)$ & $<0.0001$ & $1.17(1.05-1.30)$ & 0.003 \\
\hline Post-LVEF (\%, per unit change) & $0.99(0.97-1.00)$ & 0.29 & & \\
\hline
\end{tabular}

Chi-square for the overall multivariate model was $54.82, p<0.0001$. XRT indicates mediastinal radiation exposure

STS Society of Thoracic Surgeons, BMI body mass index, CAD coronary artery disease, COPD chronic obstructive pulmonary disease, ICM ischemic cardiomyopathy, $P C I$ percutaneous coronary intervention, $H b$ hemoglobin, $L V E F$ left ventricular ejection fraction, AVA aortic valve area, $A V$ aortic valve, $L V$-SVI left ventricular stroke volume index, RVSP right ventricular systolic pressure, $A R$ aortic regurgitation, MR mitral regurgitation, $T R$ tricuspid regurgitation, MS mitral stenosis, FEV1 forced expiratory volume at $1 \mathrm{~s}, K C C Q 12$ Kansas City Cardiomyopathy Questionnaire

study in patients with SAVR and CABG, presence of prior C-XRT was associated with worse longer-term survival [8]. Other smaller studies in prior C-XRT have also reported the presence of constrictive pericarditis, reduced pre-operative LVEF, concomitant pulmonary fibrosis, longer cardiopulmonary bypass time and hostile chest environment (radiation induced fibrosis/adhesions, and presence of multiple cardiac lesions) to be strongly associated with increased mortality [20-23]. In our study, we found no significant differences in preoperative LVEF and FEV1 in patients with prior C-XRT and hostile chest environment likely have less impact in these patients since TAVR involves percutaneous approaches. Besides, there was higher incidence of HF, AF, stroke and AV conduction abnormalities requiring PPM in the XRT subsets, which might also have contributed to the increased mortality. These data are important for prior adjudication and counseling of patients prone to develop such complications.

\section{Incidence of atrial fibrillation, stroke, heart failure and conduction abnormalities}

We noted higher incidence of AF in XRT cohorts after TAVR. Prior study from Siregar and associates showed increased incidence of AF after cardiac surgery in Hodgkin's lymphoma survivors with history of C-XRT [24]. Other studies have also demonstrated a higher prevalence of AF in patients with history of cancer. One plausible explanation for this observation is the presence of shared risk factors for $\mathrm{AF}$ and cancer including age, higher body mass index, hypertension and history of smoking [25]. There is evidence that radiation induces a low-grade inflammation in the myocardium which can lead to progressive interstitial fibrosis [26]. Presence of inflammation and fibrosis in the atrial tissues can increase the propensity to develop AF. Ascertainment of the mechanisms of increased incidence of AF in these patients will probably need cardiac MRI with comprehensive tissue characterization or histological analysis of the affected cardiac tissue.

Additionally, patients with prior C-XRT showed nearly double the incidence of stroke post-TAVR. The incidence of stroke in our patients without prior C-XRT was identical to previously reported incidence at 3-6\% [27]. Patients with prior C-XRT also had higher incidence of $\mathrm{AF}$, which might have contributed to the increased incidence of stroke, but it's also plausible that these patients have higher incidence of atherosclerosis and aortic calcifications that are known to increase the propensity for stroke with percutaneous vessel manipulation during TAVR $[14,28,29]$. With increasing use of distal protective devices during TAVR, the incidence of stroke, particularly related to atheroma breakdown, is expected to decline [30]. Moreover, patients with Hodgkin's lymphoma had higher incidence of stroke compared to other cancer subtypes which may relate to increased risk of atherosclerosis from higher dose of mediastinal radiation on the large arteries. Future prospective studies will need to address whether oral anticoagulation is beneficial in these patients.

The incidence of HF hospitalization was almost double in the XRT group (nearly 31\%) despite no differences in their baseline LVEF. Prior studies have demonstrated that nearly a quarter of the patients return to hospital 
within a year due to HF post-TAVR [31]. A seminal study by Durand and associates reported that pre-TAVR low aortic mean gradient, left atrial dilation, postprocedure anemia requiring blood transfusion and persistent severe pulmonary hypertension post-TAVR were associated with increased incidence of HF hospitalization [31]. In our XRT group, aortic mean gradients before and after TAVR, and right ventricular systolic pressure at baseline were similar compared to non-XRT group. However, the incidence of anemia and need for blood transfusion was higher in XRT group which is consistent with earlier findings and might have played a contributory role for cardiac decompensation. It is also important to state the role of diastolic function in HF patients with C-XRT since it is known to induce myocardial fibrosis perpetuating diastolic dysfunction, which was not completely addressed in our study [26, 32].

The incidence of PPM implant in our patients undergoing TAVR was $\sim 10 \%$. Notably, patients with C-XRT had double the incidence $(\sim 20 \%)$ of conduction abnormalities requiring PPM. While the exact mechanisms for these conduction abnormalities are not well understood, these are likely contributed by microvascular damage, ischemia of the conducting myocytes or direct injury of the sinoatrial node, atrioventricular node and conducting myocytes [32]. This is an important finding since TAVR procedure in itself has higher incidence of high-grade conduction abnormalities compared to SAVR with need for PPM approaching up to $25 \%$ in some studies. In patients with prior C-XRT, a close monitoring is needed to detect and treat these life-threating conduction diseases [33].

\section{Gender disparity within C-XRT TAVR}

We noted higher incidence of all-cause mortality in male counterparts compared to females who had prior C-XRT but no significant differences in MACE or cardiac mortality. Though males had higher prevalence of underlying CAD and lower pulmonary functional capacity at baseline, females had more anginal symptoms compared to male counterparts who had prior chest XRT. Previous studies by Chandrasekhar et al. and Hayashida et al. showed that females had better survival at 1 year after TAVR compared to male counterparts $[34,35]$. Thus, it is important to note that outcomes of TAVR based on gender analysis is similar in patients with prior C-XRT versus general population undergoing TAVR and male had overall worse outcomes. The effects of chest radiation on gender in these subsets of patients are unknown and warrant further studies.

\section{Quality of life}

The overall baseline functional status of all the patients was poor based on KCCQ-12 score categorization, with a trend towards a very poor category in patients with prior C-XRT [36]. The overall functional status seemed similar to previously reported data from the TVT registry [37]. On average, post TAVR, there were similar improvements in both the groups by more than average of 40 points in the KCCQ12 scores at 30 days and at 1-year after TAVR which are slightly higher than previously reported in clinical trials or data from TVT registry [3638]. Our study also shows that patients with lower preprocedure and smaller improvement of the KCCQ-12 score post-TAVR are likely to have higher mortality. This finding can further guide the clinicians in selecting patients who can benefit from TAVR and help prognosticating the outcomes after TAVR.

\section{Limitations}

We acknowledge the following limitations. First, our study is an observational cohort study and lacks the inherent strength of a randomized controlled trial. However, this study provides important insight into the natural history of TAVR for symptomatic severe aortic stenosis patients with prior history of C-XRT. Second, there remains the concern for an adequate capture of clinical outcomes mainly resulting from lack of formal follow-up beyond 12-months. For patients who did not follow up clinically in our health-care system, we obtained their data by shared clinical chart review and standardized telephone interview. Besides, the trends of primary and secondary events separated significantly early between the two groups and continued to widen over the duration of follow-up which suggests a potentially even greater magnitude of difference over time. Third, our study was not able to specify the radiation dose and adjuvant chemotherapy regimens which might have impacted the cardiovascular outcomes. We know that anthracyclines can cause cardiotoxicity leading to cardiomyopathy and heart failure, but antracyclines are not known to cause valvular stenosis. The effect of additional adjuvant regimens in patients with prior C-XRT undergoing TAVR is undefined and needs further study [39] [40].,

\section{Conclusions}

Based on this observational study, we conclude that patients with prior history of chest radiation treatment are at higher risk of adverse cardiovascular events and poor survival after TAVR. These patients may require more robust screening, follow up and clinical vigilance as they tend to do worse than general population undergoing TAVR. These data will also help in pre-procedure counselling of these patients.

\section{Additional files}

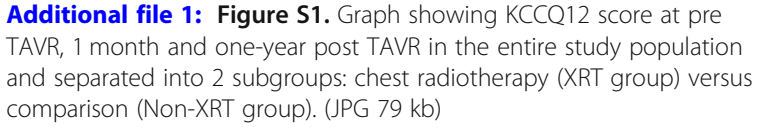

Additional file 1: Figure S1. Graph showing KCCQ12 score at pre TAVR, 1 month and one-year post TAVR in the entire study population and separated into 2 subgroups: chest radiotherapy (XRT group) versus comparison (Non-XRT group). (JPG 79 kb) 
Additional file 2: Figure S2. Graph showing KCCQ12 score at pre TAVR, 1 month and one-year post TAVR in the entire study population and separated into 2 subgroups: surviving vs non-surviving groups). * denotes statistically significant differences among the groups. (JPG $87 \mathrm{~kb}$ )

Additional file 3: Table S1. Clinical Outcomes in Relation to Types of Malignancies Treated with lonizing Radiation. (DOCX 16 kb)

\section{Abbreviations}

AF: Atrial fibrillation; AMl: Acute myocardial infarction; AV: Atrioventricular; HF: Heart Failure; KCCQ-12: Quality of Life Questionnaire; MACE: Major adverse cardiac events; PPM: Permanent pacemaker; RIVHD: RadiationInduced Valvular Heart Disease; XRT: Thoracic ionizing radiation

\section{Acknowledgements}

None.

\section{Authors' contributions}

NA and SK participated in retrieval of the clinical datasets, data analysis and manuscript writing. SW, AK, VS, AS participated in the additional analyses of clinical imaging data and retrieval of follow up data. BJP, VI and SP provided conceptual ideas and supervised the data analysis and interpretation process. KMA performed statistical analysis and ensured the rigor of the analytical methods. UCS was responsible for overall study design, mentorship, manuscript preparation and funding this study. All authors read and approved the final manuscript.

\section{Funding}

This research was supported by the National Center for Advancing Translational Sciences of the National Institutes of Health (award number UL1TR001412) to the University at Buffalo. Dr. Sharma is supported by Mentored Career Development Award from the NIH/NHLBI 5 K08 HL13198702. Dr. Pokharel and Dr. Attwood are supported by Roswell Park Comprehensive Cancer Center and National Cancer Institute (NCl) grant P30CA016056

\section{Availability of data and materials}

The published data will be made available upon satisfactory written request.

\section{Ethics approval and consent to participate}

All clinical procedures and protocols conformed to institutional guidelines and were approved by the Institutional review board (IRB) at University at Buffalo, Buffalo, NY.

\section{Consent for publication}

Not applicable.

\section{Competing interests}

The authors declare that they have no competing interests.

\section{Author details}

'Department of Medicine, Division of Cardiology, Clinical \& Translational Research Center (Suite 7030), Jacob's School of Medicine and Biomedical Sciences, Buffalo, NY, USA. Department of Radiology, Brigham and Women's Hospital, Boston, MA, USA. ${ }^{3}$ Department of Biostatistics and Bioinformatics, Roswell Park Comprehensive Cancer Center, Buffalo, NY, USA. ${ }^{4}$ Department of Pathology, Division of Thoracic Pathology and Oncology, Roswell Park Comprehensive Cancer Center, Buffalo, NY, USA. ${ }^{5}$ Clinical \& Translational Research Center (Suite 7030), 875 Ellicott Street, Buffalo, NY 14203, USA.

\section{Received: 22 April 2019 Accepted: 26 June 2019}

\section{Published online: 22 July 2019}

\section{References}

1. Mahmood SS, Nohria A. Cardiovascular complications of cranial and neck radiation. Curr Treat Options Cardiovasc Med. 2016;18(7):45.

2. Nabialek-Trojanowska I, Lewicka E, Wrona A, et al. Cardiovascular complications after radiotherapy. Cardiol J. 2018. https://doi.org/10.5603/CJ. a2018.0120.

3. Gustavsson A, Osterman B, Cavallin-Stahl E. A systematic overview of radiation therapy effects in non-Hodgkin's lymphoma. Acta Oncol. 2003;42(5-6):605-19.
4. Armanious MA, Mohammadi H, Khodor S, Oliver DE, Johnstone PA, Fradley MG. Cardiovascular effects of radiation therapy. Curr Probl Cancer. 2018;42(4):433-42.

5. Copeland KA, Hosmane VR, Jurkovitz C, et al. Frequency of severe valvular disease caused by mediastinal radiation among patients undergoing valve surgery in a community-based, regional academic medical center. Clin Cardiol. 2013;36(4):217-21.

6. Mousavi N, Nohria A. Radiation-induced cardiovascular disease. Curr Treat Options Cardiovasc Med. 2013;15(5):507-17.

7. Bouillon K, Haddy N, Delaloge S, et al. Long-term cardiovascular mortality after radiotherapy for breast cancer. J Am Coll Cardiol. 2011;57(4):445-52.

8. Donnellan E, Masri A, Johnston DR, et al. Long-term outcomes of patients with mediastinal radiation-associated severe aortic stenosis and subsequent surgical aortic valve replacement: a matched cohort study. J Am Heart Assoc. 2017;6(5). https://doi.org/10.1161/JAHA.116.005396.

9. Carver JR, Shapiro CL, Ng A, et al. American Society of Clinical Oncology clinical evidence review on the ongoing care of adult cancer survivors: cardiac and pulmonary late effects. J Clin Oncol. 2007;25(25): 3991-4008.

10. Jaworski C, Mariani JA, Wheeler G, Kaye DM. Cardiac complications of thoracic irradiation. J Am Coll Cardiol. 2013;61(23):2319-28.

11. Adams MJ, Hardenbergh PH, Constine LS, Lipshultz SE. Radiation-associated cardiovascular disease. Crit Rev Oncol Hematol. 2003;45(1):55-75.

12. Wethal $T$, Lund MB, Edvardsen $T$, et al. Valvular dysfunction and left ventricular changes in Hodgkin's lymphoma survivors. A longitudinal study. Br J Cancer. 2009:101(4):575-81.

13. Tamura A, Takahara Y, Mogi K, Katsumata M. Radiation-induced valvular disease is the logical consequence of irradiation. Gen Thorac Cardiovasc Surg. 2007;55(2):53-6.

14. Hull MC, Morris CG, Pepine CJ, Mendenhall NP. Valvular dysfunction and carotid, subclavian, and coronary artery disease in survivors of hodgkin lymphoma treated with radiation therapy. JAMA. 2003:290(21):2831-7.

15. Waksman R, Rogers T, Torguson R, et al. Transcatheter aortic valve replacement in low-risk patients with symptomatic severe aortic stenosis. J Am Coll Cardiol. 2018;72(18):2095-105.

16. Lang RM, Badano LP, Mor-Avi V, et al. Recommendations for cardiac chamber quantification by echocardiography in adults: an update from the American Society of Echocardiography and the European Association of Cardiovascular Imaging. Eur Heart J Cardiovasc Imaging. 2015;16(3):233-70.

17. Baumgartner $\mathrm{H}$, Hung J, Bermejo J, et al. Echocardiographic assessment of valve stenosis: EAE/ASE recommendations for clinical practice. J Am Soc Echocardiogr. 2009;22(1):1-23; quiz 101-102.

18. Schulman S, Kearon C. Subcommittee on control of anticoagulation of the S, standardization Committee of the International Society on T, Haemostasis Definition of major bleeding in clinical investigations of antihemostatic medicinal products in non-surgical patients. J Thromb Haemost. 2005;3(4): 692-4.

19. Schulman S, Angeras U, Bergqvist D, et al. Definition of major bleeding in clinical investigations of antihemostatic medicinal products in surgical patients. J Thromb Haemost. 2010;8(1):202-4.

20. Handa N, McGregor CG, Danielson GK, et al. Valvular heart operation in patients with previous mediastinal radiation therapy. Ann Thorac Surg. 2001:71(6):1880-4

21. Hancock SL, Tucker MA, Hoppe RT. Factors affecting late mortality from heart disease after treatment of Hodgkin's disease. JAMA. 1993:270(16):1949-55.

22. Chang AS, Smedira NG, Chang CL, et al. Cardiac surgery after mediastinal radiation: extent of exposure influences outcome. J Thorac Cardiovasc Surg. 2007;133(2):404-13.

23. Desai MY, Karunakaravel K, Wu W, et al. Pulmonary fibrosis on multidetector computed tomography and mortality in patients with radiation-associated cardiac disease undergoing cardiac surgery. J Thorac Cardiovasc Surg. 2014 148(2):475-481 e473.

24. Siregar $S$, de Heer F, van Herwerden LA. Cardiac surgery in patients irradiated for Hodgkin's lymphoma. Neth Heart J. 2010;18(2):61-5.

25. O'Neal WT, Lakoski SG, Qureshi W, et al. Relation between cancer and atrial fibrillation (from the REasons for geographic and racial differences in stroke study). Am J Cardiol. 2015;115(8):1090-4.

26. El-Sherif O, Xhaferllari I, Sykes J, et al. (18) F-FDG cardiac PET inflammation imaging in a canine model of radiation induced cardiac disease associated with breast cancer radiotherapy. Am J Physiol Heart Circ Physiol. 2019:316(3): H586-H595. https://doi.org/10.1152/ajpheart.00273.2018. Epub 2018 Dec 21. 
27. Ahmad Y, Demir O, Rajkumar C, et al. Optimal antiplatelet strategy after transcatheter aortic valve implantation: a meta-analysis. Open Heart. 2018;5(1):e000748.

28. Reed GW, Masri A, Griffin BP, Kapadia SR, Ellis SG, Desai MY. Long-term mortality in patients with radiation-associated coronary artery disease treated with percutaneous coronary intervention. Circ Cardiovasc Interv. 2016;9(6). https://doi.org/10.1161/CIRCINTERVENTIONS.115.003483.

29. Daitoku K, Fukui K, Ichinoseki I, Munakata M, Takahashi S, Fukuda I. Radiotherapy-induced aortic valve disease associated with porcelain aorta. Jpn J Thorac Cardiovasc Surg. 2004;52(7):349-52.

30. Demir OM, lannopollo G, Mangieri A, et al. The role of cerebral embolic protection devices during Transcatheter aortic valve replacement. Front Cardiovasc Med. 2018:5:150.

31. Durand E, Doutriaux M, Bettinger N, et al. Incidence, prognostic impact, and predictive factors of readmission for heart failure after Transcatheter aortic valve replacement. JACC Cardiovasc Interv. 2017;10(23):2426-36.

32. Taunk NK, Haffty BG, Kostis JB, Goyal S. Radiation-induced heart disease: pathologic abnormalities and putative mechanisms. Front Oncol. 2015;5:39.

33. Maan A, Refaat MM, Heist EK, et al. Incidence and predictors of pacemaker implantation in patients undergoing Transcatheter aortic valve replacement. Pacing Clin Electrophysiol. 2015;38(7):878-86.

34. Chandrasekhar J, Dangas G, Yu J, et al. Sex-based differences in outcomes with Transcatheter aortic valve therapy: TVT registry from 2011 to 2014. J Am Coll Cardiol. 2016:68(25):2733-44.

35. Hayashida K, Morice MC, Chevalier B, et al. Sex-related differences in clinical presentation and outcome of transcatheter aortic valve implantation for severe aortic stenosis. J Am Coll Cardiol. 2012;59(6):566-71.

36. Arnold SV, Spertus JA, Lei Y, et al. Use of the Kansas City cardiomyopathy questionnaire for monitoring health status in patients with aortic stenosis. Circ Heart Fail. 2013;6(1):61-7.

37. Arnold SV, Spertus JA, Vemulapalli S, et al. Quality-of-life outcomes after Transcatheter aortic valve replacement in an unselected population: a report from the STS/ACC Transcatheter valve therapy registry. JAMA Cardiol. 2017;2(4):409-16.

38. Arnold SV, Reynolds MR, Lei Y, et al. Predictors of poor outcomes after transcatheter aortic valve replacement: results from the PARTNER (placement of aortic Transcatheter valve) trial. Circulation. 2014:129(25):2682-90.

39. Chung WB, Youn HJ. Pathophysiology and preventive strategies of anthracycline-induced cardiotoxicity. Korean J Intern Med. 2016:31(4):625-33.

40. Fukuda A, Tahara K, Hane Y, et al. Comparison of the adverse event profiles of conventional and liposomal formulations of doxorubicin using the FDA adverse event reporting system. PLoS One. 2017;12(9):e0185654.

\section{Publisher's Note}

Springer Nature remains neutral with regard to jurisdictional claims in published maps and institutional affiliations.

Ready to submit your research? Choose BMC and benefit from:

- fast, convenient online submission

- thorough peer review by experienced researchers in your field

- rapid publication on acceptance

- support for research data, including large and complex data types

- gold Open Access which fosters wider collaboration and increased citations

- maximum visibility for your research: over $100 \mathrm{M}$ website views per year

At $\mathrm{BMC}$, research is always in progress.

Learn more biomedcentral.com/submissions 